\title{
Monitoramento da carga interna no basquetebol
}

\author{
Monitoring internal load in basketball
}

\author{
João Antônio Nunes \\ Eduardo Caldas Costa 2,3 \\ Luis Viveiros 2,4 \\ Alexandre Moreira \\ Marcelo Saldanha Aoki
}

1. Universidade de São Paulo, Escola de Educação Física e Esporte. São Paulo, SP, Brasil

2. Universidade de São Paulo. Escola de Artes, Ciências e Humanidades. Grupo de Adaptações Biológicas ao Exercício Físico. São Paulo, SP, Brasil

3. Universidade Federal do Rio Grande do Norte, Natal, RN, Brasil.

4. Comitê Olímpico Brasileiro, Rio de Janeiro, RJ. Brasil

Recebido em 02/03/10 Revisado em 17/06/10 Aprovado em 27/07/10
Resumo - O objetivo do presente estudo foi quantificar a magnitude da carga interna referente a uma partida oficial de Basquetebol feminino e avaliar se a carga interna apresenta relação com o número de ações técnicas realizadas no jogo. A amostra foi constituída de 10 atletas (25 \pm 4 anos) que participaram do XI Campeonato Brasileiro de Basquetebol Feminino. Foram avaliados o comportamento da frequência cardíaca (FC) e a percepção subjetiva de esforço (PSE) em resposta à partida. A carga interna foi calculada pelo método de Edwards e pelo método de Foster. Além disso, foram analisados os parâmetros de desempenho técnico na partida. A carga interna estimada pelo método de Edwards e Foster foram $255 \pm 62$ e $321 \pm 127$ unidades arbitrárias, respectivamente. Foi detectada correlação moderada entre os dois métodos utilizados (Edwards e Foster; $r=0,64 ;-p<0,05$ ). Também foram detectadas correlações significativas $(p<0,05)$ entre a carga interna estimada pelo método de Edwards e o número de pontos marcados na partida $(\mathrm{r}=0,53)$, o número de rebotes $(r=0,67)$, o número de assistências $(r=0,59)$, o número de recuperações de posse de bola $(r=0,70)$ e o somatório de todas as ações $(r=0,64)$. Os resultados obtidos no presente estudo sugerem que a carga interna da partida avaliada foi moderada e que o número de ações realizadas pelas atletas na partida influencia a carga interna.

Palavras-chave: Percepção subjetiva do esforço; Frequência cardíaca; Basquetebol.

Abstract - The aim of this study was to quantify the magnitude of the internal load induced by an official women's basketball match and to determine whether the magnitude of the internal load is correlated with the number of basketball maneuvers perfomed. The sample consisted of 10 athletes (25 \pm 4 years), who participated in the XI Brazilian Women's Basketball Championship. Heart rate and rating of perceived exertion in response to the match were evaluated. The internal load was calculated by the methods of Edwards and Foster. In addition, the basketball maneuvers perfomed in the match were analyzed. The internal load estimated by the methods of Edwards and Foster was $255 \pm 62$ and $321 \pm 127$ arbitrary units, respectively. A moderate correlation was observed between the two methods used $(r=0.64, p<0.05)$. In addition, significant correlations $(p<0.05)$ were observed between internal load estimated by the method of Edwards and the number of points scored in the match $(r=0.53)$, number of rebounds $(r=$ $0.67)$, number of assists $(r=0.59)$, number of steals $(r=0.70)$, and the sum of all indicators $(r$ $=0.64$ ). The results of this study suggest that the internal load of the match was moderate and was influenced by the number of maneuvers performed by the athletes in the match.

Key words: Rating of perceived exertion; Heart rate; Basketball 


\section{INTRODUÇÃO}

O Basquetebol é caracterizado como um esporte de oposição e cooperação, que envolve ações simultâneas de ataque e defesa entre duas equipes ${ }^{1}$. As demandas do Basquetebol exigem grande diversidade de movimentos/ações (ex. arremessos, rebotes, ataque, defesa e contra-ataque) e rápidas transições entre o ataque e a defesa ${ }^{1-3}$. Sendo assim, o Basquetebol é classificado como um esporte de natureza intermitente ${ }^{4}$. Esse padrão de atividade constituído por esforços intermitentes de alta intensidade impõe significativa sobrecarga física sobre os jogadores 5 .

As exigências do Basquetebol (carga externa) afetam o equilíbrio do meio interno, promovendo alterações (carga interna) em parâmetros como: a concentração de lactato, a frequência cardíaca (FC) e a percepção subjetiva do esforço (PSE) ${ }^{1,2,6-}$ ${ }^{8}$. No entanto, é importante ressaltar que existem poucos dados disponíveis sobre a quantificação da carga interna em partidas oficiais. A investigação mais recente conduzida para verificar as respostas decorrentes de jogos oficiais no Basquetebol, após as significativas alterações nas regras, foi realizada por Ben Abdelkrim et al. ${ }^{1}$.

O estudo de Ben Abdelkrim et al. ${ }^{1}$ avaliou o impacto das novas regras (a partir de maio de 2000) sobre sobrecarga interna em jogos de Basquetebol. Os achados do referido estudo revelaram valores de FC superiores aos encontrados por McInees et al. ${ }^{2}$ na década de 90. Apesar dos estudos acima citados, ainda é possível afirmar que pouco se conhece sobre a carga interna associada aos jogos oficiais.

Em esportes de caráter intermitente, como o Basquetebol, é difícil avaliar/quantificar a carga interna imposta aos atletas ${ }^{911}$. Entretanto, alguns métodos têm sido utilizados com intuito de monitorar as cargas de treinamento em atividades intermitentes. Esses métodos de quantificação da carga interna são baseados no comportamento da FC, mais especificamente, o tempo dispendido em diferentes zonas de $\mathrm{FC}^{11-13}$. Essas metodologias têm sido utilizadas, tanto em sessões de treinamento, quanto em competições ${ }^{11,13,14}$. $\mathrm{O}$ método proposto por Edwards ${ }^{12}$ é um dos mais populares, sendo utilizado em esportes intermitentes, como o Futebol e o Basquetebol ${ }^{6,15,16}$.

O referido método quantifica a carga interna através do tempo dispendido (em minutos) em cinco zonas diferentes de $\mathrm{FC} \mathrm{(50-60 \%} \mathrm{da} \mathrm{FC}_{\text {máx }}=$ zona 1; $>60-70 \%$ da $\mathrm{FC}_{\text {máx }}=$ zona $2 ;>70-80 \%$ da $\mathrm{FC}_{\max }=$ zona $3 ;>80-90 \%$ da $\mathrm{FC}_{\max }=$ zona $4 ;>90-100 \%$ da
$\mathrm{FC}_{\text {máx }}=$ zona 5). $\mathrm{O}$ tempo dispendido (minutos) em cada zona (1,2,3,4 e 5) é multiplicado pelo número referente à mesma. Por exemplo, uma sessão de treino com duração de 60 minutos, sendo 30 minutos na zona 2 e 30 minutos na zona 3 , terá sua carga interna estimada em 150UA (unidades arbitrárias).

Outro método de quantificação da carga interna bastante simples é o Método da Percepção Subjetiva do Esforço (PSE) da sessão ${ }^{6}$. O método da PSE utiliza a pergunta: "Como foi a sua sessão de treino?". A resposta deve ser fornecida a partir de uma escala adaptada da CR10 de Borg ${ }^{17}$, conforme ilustrado por Nakamura et al. ${ }^{18}$. Essa medida deve refletir uma avaliação global da intensidade de toda a sessão, e o intervalo de cerca de 30 minutos deve ser adotado para que atividades leves ou pesadas realizadas ao final da sessão não influenciem a avaliação. O produto do escore da PSE (intensidade - exemplo: 6.5) pela duração da sessão em minutos (volume - exemplo: 40) reflete a magnitude da carga interna (260UA) em unidades arbitrárias ${ }^{6,18}$.

A PSE da sessão também tem sido utilizada em outros esportes coletivos ${ }^{6,15,16,19}$. Foster et al. ${ }^{6}$ verificaram correlação significativa entre o método objetivo de Edwards ${ }^{12}$ e o método subjetivo de Foster et al. ${ }^{6}$ durante diferentes tipos de atividade com caráter intermitente, sugerindo que ambos podem ser utilizados em atividades dessa natureza para quantificar a carga interna. Conforme já mencionado anteriormente, os estudos relacionados à quantificação da carga interna de partidas oficiais de Basquetebol são escassos. Essa escassez é ainda maior para o Basquetebol feminino. O conhecimento da sobrecarga interna da atividade competitiva é fundamental para maximizar o efeito das estratégias de treinamento e recuperação, visando o incremento do desempenho.

Diante do exposto, o objetivo do presente estudo foi quantificar a magnitude da carga interna referente a uma partida oficial de Basquetebol feminino. Além disto, este estudo investigou a hipótese de que magnitude da carga interna apresenta relação com as ações técnicas de jogo.

\section{PROCEDIMENTOS METODOLÓGICOS}

\section{Delineamento experimental}

A coleta de dados foi realizada durante uma partida do Campeonato Brasileiro de Basquetebol Feminino. Na referida partida, foram monitorados o comportamento da FC, a PSE da sessão e as ações técnicas de jogo desempenhadas pelas atletas. 


\section{Amostra}

A amostra do presente estudo foi constituída por 10 atletas ( $25 \pm 4$ anos; $179 \pm 8 \mathrm{~cm} \mathrm{e} 73 \pm 9 \mathrm{~kg}$ ) que participaram do XI Campeonato Brasileiro de Basquetebol Feminino - 2009. Anteriormente ao processo de coleta dos dados, as atletas foram esclarecidas em relação aos objetivos e procedimentos da pesquisa. As atletas concordaram em participar, voluntariamente, do procedimento experimental, assinando um termo de consentimento livre e esclarecido. $O$ procedimento experimental foi aprovado pelo Comitê de Ética em Pesquisa da Escola de Educação Física e Esporte da Universidade de São Paulo, processo n ${ }^{0}$ 2008/37.

Mensuração da freqüência cardíaca máxima O comportamento da FC das atletas foi avaliado através do equipamento Polar Team System $\left(\operatorname{Polar}^{\circledR}\right)$ que permitiu estratificar o tempo total acumulado em cada uma das cinco zonas de referências $-50-60 \%$, > 60-70\%, > 70-80\%, > 80-90\% e $>90-100 \%$ da FC máxima - como sugerido por Edwards $^{12}$. O cálculo referente à estimativa da carga interna foi realizado a partir do produto do tempo acumulado em cada zona de FC por seu número correspondente $(50-60 \%=1 ;>60-70 \%=$ $2 ;>70-80 \%=3 ;>80-90 \%=4 ;>90-100 \%=5)$. O somatório de todas as zonas foi utilizado para determinar a magnitude da carga interna.

\section{Mensuração da percepção subjetiva de esforço}

A quantificação da carga interna também foi determinada através do método da PSE da sessão ${ }^{6}$. O método da PSE utiliza a pergunta: "Como foi a sua sessão de treino?". No referido método, a resposta deve ser fornecida 30 minutos após o término da sessão de treino, a partir da escala adaptada CR10 de $\mathrm{Borg}^{17}$. O avaliador deve instruir o avaliado a escolher o descritor e depois o número de 0 a 10 , que pode ser fornecido em decimais (por exemplo: 7,5). O valor máximo (10) deve ser comparado ao maior esforço físico experimentado pela pessoa e o valor mínimo é a condição de repouso absoluto (zero). Neste método, o escore da PSE equivale a intensidade da atividade e a duração da atividade representa o volume. $\mathrm{O}$ produto da intensidade (PSE $=6,5)$ pelo volume (90 minutos) expressa a magnitude da carga interna (585 UA) em unidades arbitrárias.

\section{Análise dos indicadores de desempenho de jogo}

A análise dos indicadores de desempenho de jogo foi realizada seguindo os critérios padronizados pela Confederação Brasileira de Basquetebol (CBB). A análise dos jogos do campeonato nacional é realizada por 3 membros da comissão de estatística da CBB, com auxílio de software específico. Os indicadores de desempenho de jogo utilizados no presente estudo foram: tempo de jogo, quantidade de arremessos realizados ( 3 pts, 2 pts e lances-livres), quantidade de arremessos convertidos ( 3 pts, 2 pts e lances-livres), rebotes de defesa e de ataque, assistências e bolas recuperadas.

\section{Análise estatística}

Os dados foram expressos em média \pm desvio padrão. A fim de testar a hipótese da existência de correlação entre os métodos de monitoramento da carga de treinamento, foram determinados os coeficientes de correlação de Pearson. Também foram calculados os coeficientes de correlação entre a carga de treinamento e os indicadores de desempenho de jogo. No presente estudo, foi adotada a classificação de faixas de correlação sugerida por Giannichi ${ }^{20}$. Este autor considera uma correlação fraca entre 0 e 0,59; moderada entre 0,60 e 0,79; boa entre 0,80 e 0,89 e excelente entre 0,90 e 1. Foi realizada uma ANOVA de medidas repetidas para comparar a magnitude das sessões de treinamento na semana do jogo investigado. Quando necessário, o teste post-hoc de Bonferroni foi utilizado. $\mathrm{O}$ nível de significância adotado foi de 5\%. O nível de significância estatística adotado foi de 5\%.

\section{RESULTADOS}

A quantificação da carga interna estimada pelo método de Edwards ${ }^{12}(255 \pm 62 \mathrm{UA})$ e pelo método de Foster et al. ${ }^{6}(321 \pm 127 \mathrm{UA})$ sugere que a sobrecarga da partida foi moderada ${ }^{21}$. O valor médio da PSE reportada pelas atletas $(3,9 \pm 1,5)$ reforça a concepção de que a intensidade relacionada à partida foi moderada (Figura 1).

Os resultados observados na figura 2 reforçam a existência de correlação moderada entre o método proposto por Edwards ${ }^{12}$ (1993) e o método de Foster et al. ${ }^{6}$ no que se refere à quantificação da carga interna $(r=0,64-p<0,05)$.

A figura 3 ilustra a distribuição percentual do tempo total de jogo em relação às zonas de intensidade propostas por Edwards ${ }^{12}$. A determinação do valor médio das 10 atletas permitiu que a distribuição do tempo total fosse estabelecida: 19,3\% na zona $5,21,2 \%$ na zona $4,17,5 \%$ na zona $3,14,7 \%$ na zona 2 e $27,3 \%$ na zona 1 . 


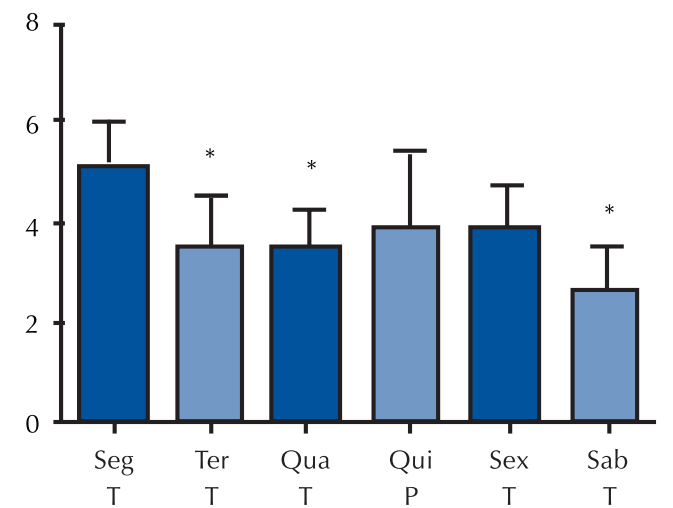

* - p>0,05 em relação à sessão de treino realizada na segunda-feira (Seg).

Figura 1. Intensidade referente às sessões de treinamento $(T)$ e à partida (P), aferida pelo método da PSE da sessão.

$$
r=0.64 ; p=0.04
$$

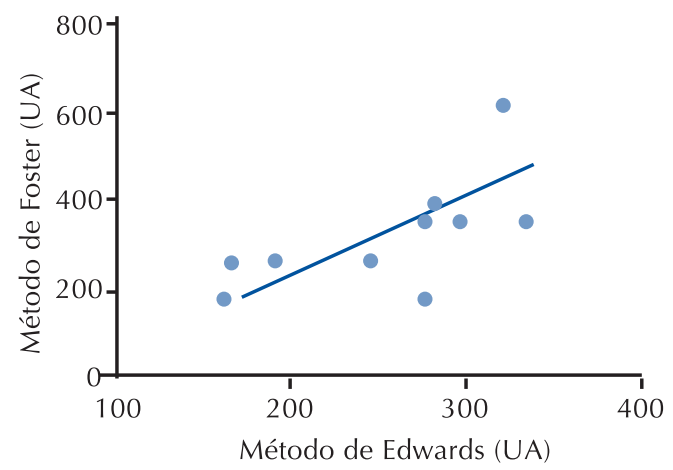

Figura 2. Correlação entre a carga interna estimada pelo método PSE da sessão e a carga interna estimada pelo método de Edwards.

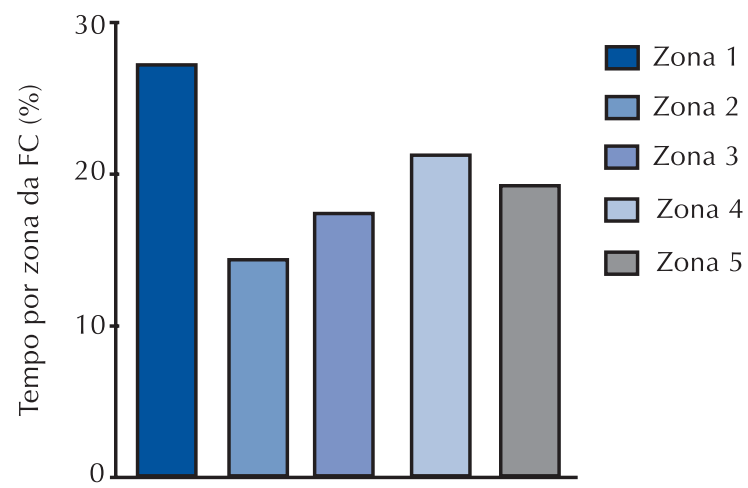

Figura 3. Distribuição do tempo total da partida por zona de frequência cardíaca.
A tabela 1 mostra a correlação entre a carga interna e as ações técnicas de jogo. Foram detectadas correlações entre a carga interna estimada pelo método de Edwards ${ }^{12}$ e os pontos totais marcados na partida $(\mathrm{r}=0,53-p<0,05)$, o número de rebotes $(\mathrm{r}=0,67-p<0,05)$, o número de assistências ( $\mathrm{r}$ $=0,59-p<0,05)$, o número de recuperações de posse de bola $(\mathrm{r}=0,70-p<0,05)$ e o somatório de todas as ações $(r=0,64-p<0,05)$, respectivamente (Tabela 1). Foi encontrada correlação fraca $(\sim \mathrm{r}=$ $0,4)$ entre a carga interna estimada pelo método PSE da sessão e os parâmetros supracitados (dados não reportados).

\section{DISCUSSÃO}

Este é o primeiro relato sobre quantificação da carga interna referente a uma partida oficial do Campeonato Brasileiro de Basquetebol Feminino. O presente estudo também investigou a relação entre a magnitude da carga interna e os indicadores de desempenho de jogo.

O método proposto por Edwards ${ }^{12}$ parece ser adequado para quantificação da carga interna em atividades e/ou esportes intermitentes, pois o mesmo considera o tempo gasto (em minutos) em cinco zonas diferentes de FC, em um continuum de leve a máxima, para determinação da sobrecarga interna ${ }^{6,12,22}$. Este método constitui uma alternativa para a avaliação da carga interna no Basquetebol ${ }^{6}$, considerando as características intermitentes dessa modalidade.

Já é sabido que, aproximadamente, $15 \%$ das ações realizadas durante uma partida ocorrem em alta intensidade ${ }^{1,2}$. Além disso, durante o jogo há constantes mudanças de direção ( 1.000 por partida $)^{2}$, repetidos momentos de aceleração/desaceleração $0^{2,3}$ e saltos diversos ${ }^{3}$, gerando elevada carga interna para os atletas ${ }^{5}$.

Nesse sentido, a utilização de um método objetivo para quantificação da carga interna, como

Tabela 1. Correlação entre a carga interna estimada pelo método de Edwards (UA) e as ações técnicas de jogo.

\begin{tabular}{|c|c|c|c|c|c|c|}
\hline Atletas & Carga & Pontos & Rebotes & Recuperações & Assistências & Total \\
\hline 1 & 162 & 0 & 0 & 0 & 0 & 0 \\
\hline 2 & 277 & 2 & 3 & 1 & 1 & 7 \\
\hline 3 & 297 & 21 & 4 & 3 & 5 & 33 \\
\hline 4 & 192 & 9 & 2 & 0 & 1 & 12 \\
\hline 5 & 282 & 14 & 2 & 2 & 2 & 20 \\
\hline 6 & 334 & 11 & 3 & 1 & 3 & 18 \\
\hline 7 & 167 & 0 & 0 & 0 & 0 & 0 \\
\hline 8 & 277 & 5 & 0 & 0 & 0 & 5 \\
\hline 9 & 246 & 7 & 4 & 0 & 1 & 12 \\
\hline \multirow[t]{2}{*}{10} & 322 & 5 & 5 & 1 & 3 & 14 \\
\hline & & $r=0,53^{*}$ & $r=0,67^{*}$ & $r=0,59^{*}$ & $r=0,70^{*}$ & $r=0,64^{*}$ \\
\hline
\end{tabular}

$*-p<0,05$. 
o proposto por Edwards ${ }^{12}$, pode esclarecer aspectos relevantes, do ponto da sobrecarga fisiológica, que ocorrem durante uma partida oficial (ex. tempo gasto em zonas leve, moderada, intensa e máxima de FC). $\mathrm{O}$ conhecimento bem detalhado sobre o tempo dispendido em cada zona de FC durante o jogo pode, inclusive, nortear estratégias de treinamento, priorizando o maior nível de especificidade no que se refere à carga interna imposta aos atletas em uma partida oficial.

Rodriguez-Alonso et al. ${ }^{7}$ verificaram em partida oficial de Basquetebol feminino, altos valores de FC para armadoras (185 bpm), alas (175 bpm) e pivôs (167 bpm). Os resultados do presente estudo corroboram o padrão encontrado pelos autores supracitados, sendo que foram observados valores de $\mathrm{FC}_{\text {máx }}$ de 202, 192 e 189 bpm para armadoras $(n=2)$, alas $(n=4)$ e pivôs $(n=4)$, respectivamente, durante a partida analisada. Entretanto, Rodriguez-Alonso et al. ${ }^{7}$ apenas descreveram o comportamento da FC, não utilizando métodos de quantificação da carga interna. É plausível assumir que o conhecimento da distribuição estratificada da FC por zonas de leve a máxima, como proposto por Edwards $^{12}$, pode ser mais adequado (do que o valor médio ou de pico) para determinar a magnitude da carga interna imposta aos atletas.

Entretanto, pouco se sabe sobre o comportamento da FC durante uma partida oficial no tocante ao tempo relativo (\%) que os atletas permanecem em cada zona. Isso ocorre, principalmente, pela dificuldade de se mensurar o tempo real jogado pelos atletas, haja vista que menos de $50 \%$ da duração da partida é, de fato, disputada ativamente ${ }^{5}$. No presente estudo, foi avaliado o comportamento da FC durante o tempo total da partida. Pode-se notar que 40\% ( 35 minutos) do tempo total é dispendido nas zonas 4 e 5 . Ao analisar apenas as atletas que jogaram mais que 20 minutos, observa-se que o tempo dispendido nas zonas 4 e 5 sofre acréscimo de $5 \%$, perfazendo $45 \%$ do tempo total. O restante do tempo ( $60 \%)$ é gasto nas zonas de menor intensidade (3, 2 e 1), o que reforça o caráter intermitente da partida.

Outra alternativa viável para a quantificação da carga interna é a utilização do método da PSE da sessão ${ }^{6}$ em competições oficiais. A PSE representa a percepção do atleta no que se refere ao estresse do treinamento e/ ou competição ${ }^{6,21}$, que pode incluir aspectos fisiológicos e psicológicos. Esta avaliação global, proposta pelo método PSE da sessão ${ }^{6}$, pode auxiliar a determinação da carga interna no ambiente competitivo.

Impellizzeri et al. ${ }^{15}$ demonstraram correlação entre o método proposto por Edwards ${ }^{12}$ e o método da PSE da sessão ${ }^{6}(r=0,71)$ no Futebol. Alexiou e Coutts $^{16}$ também verificaram o mesmo padrão de correlação em atletas de Futebol do sexo feminino $(\mathrm{r}=0,85)$. Ambos os autores sugerem que o método PSE da sessão ${ }^{6}$ pode ser utilizado nesse esporte para quantificar a carga interna imposta aos atletas. Os resultados do presente estudo indicam que existe correlação entre os métodos de Edwards ${ }^{12}$ e Foster et al. $^{6}(\mathrm{r}=0,64)$. Apesar de inferior aos valores previamente observados ${ }^{15,16,23}$, os achados do presente estudo são relevantes, principalmente, sob o ponto de vista da validade ecológica, haja vista que a quantificação da carga interna foi realizada em partida oficial, com presença de todos os componentes que envolvem a mesma.

$\bigcirc$ presente estudo corrobora os resultados reportados pelos autores supracitados, reforçando que a PSE da sessão pode ser utilizada para definir a magnitude de carga interna imposta às atletas na partida. Foster et al. ${ }^{21}$ também propuseram uma classificação de intensidade para sessões de treinamento: fácil $=$ PSE $<3$; moderada $=$ PSE entre 3-5; difícil $=$ PSE $>$ 5. No presente estudo, a média da PSE reportada pelas atletas foi de 3,9 (escore mínimo 2 e escore máximo 7), portanto, segundo Foster et $\mathrm{al}^{21}$, a intensidade relacionada à partida avaliada seria classificada como moderada. O monitoramento da intensidade demonstrou que este parâmetro foi semelhante entre as sessões de treinamento e a partida. Este dado indica que a prescrição da intensidade das cargas de treinamento foi específica em relação à condição real de jogo.

No que se refere à análise de correlação entre a carga interna e os indicadores de desempenho de jogo, os dados demonstram que o método proposto por Edwards ${ }^{12}$ apresentou correlação significante com todos os parâmetros de desempenho de jogo avaliados. Estes dados reforçam a hipótese de que quanto maior o número de ações desenvolvidas ativamente pelas atletas no jogo (número de pontos marcados, rebotes, assistências e recuperações de posse de bola), maior será a carga interna imposta as mesmas. Portanto, é possível especular que a realização de ações de jogo, que tem influência direta no resultado final da partida, está relacionada à maior carga interna.

\section{CONCLUSÃO}

Os resultados do presente estudo sugerem que a sobrecarga da partida avaliada foi moderada. Este relato também reforçou a existência de correlação entre o método proposto por Edwards e o método da 
PSE da sessão. Além disso, a carga interna estimada pelo método de Edwards apresentou correlação com os indicadores de desempenho técnico de jogo (número de pontos marcados, rebotes, assistência e recuperações de posse de bola). Estes dados indicam que o número de ações realizadas na partida pelas atletas influencia a carga interna. $\bigcirc$ monitoramento da carga interna de partidas oficiais pode auxiliar a comissão técnica no planejamento/periodização das futuras cargas de treinamento.

\section{REFERÊNCIAS BIBLIOGRÁFICAS}

1. Ben Abdelkrim N, El Fazaa S, El Ati J. Time-motion analysis and physiological data of elite under-19-year-old basketball players during competition. Br J Sports Med 2007;41:69-75.

2. McInnes SE, Carlson JS, Jones CJ, McKenna MJ. The physiological load imposed on basketball players during competition. J Sports Sci 1995;13:387-97.

3. Janeira MA, Maia J. Game intensity in basketball: An interactionist view linking time-motion analysis, lactate concentration and heart rate. Coaching and Sport Sci J 1998;3:26-30.

4. Moreira A, Oliveira PR, Ronque ERV, Okano AH, Souza M. Análise de diferentes modelos de estruturação da carga de treinamento e competição no desempenho de basquetebolistas. Rev Bras Ciênc Esporte 2008;29:165-83.

5. Drinkwater EJ, Pyne DB, McKenna MJ. Design and interpretation of anthropometric and fitness testing of basketball players. Sports Med 2008;38:565-78.

6. Foster C, Florhaug JA, Franklin J, Gottschall L, Hrovatin LA, Parker S, et al. A new approach to monitoring exercise training. J Strength Cond Res 2001;15:109-15.

7. Rodríguez-Alonso M, Fernández-García B, Pérez-Landaluce J, Terrados N. Blood lactate and heart rate during national and international women's basketball. J Sports Med Phys Fitness 2003;43:432-6.

8. Tessitore A, Tiberi M, Cortis C, Rapisarda E, Meeusen R, Capranica L. Aerobic-anaerobic profiles, heart rate and match analysis in old basketball players. Gerontology 2006;52:214-22.

9. Stagno KM, Thatcher R, van Someren KA. A modified TRIMP to quantify the in-season training load of team sport players. J Sports Sci. 2007;25:629-34.

10. Desgorces FD, Sénégas X, Garcia J, Decker L, Noirez P. Methods to quantify intermittent exercises. Appl Physiol Nutr Metab 2007;32:762-9.

11. Banister EW. Modeling elite athletic performance. In: Green HJ, McDougal JD, Wenger HA, eds. Physiological Testing of Elite Athletes. Champaign, Illinois: Human Kinetics; 1991:403-24.

12. Edwards S. High performance training and racing. In: Edwards S, editor. The Heart Rate Monitor Book. 8th ed. Sacramento, CA: Feet Fleet Press; 1993. p.113-123.
13. Lucia A, Hoyos J, Santalla A, Earnest C, Chicharro J. Tour de France versus Vuelta Espana: Which is harder? Med Sci Sports Exerc 2003;35:872-8.

14. Padilla S, Mujika J, Orbananos J, Angulo F. Exercise intensity during competition time trials in professional road cycling. Med Sci Sports Exerc 2000;32:850-6.

15. Impellizzeri FM, Rampinini E, Coutts AJ, Sassi A, Marcora SM. Use of RPE-based training load in soccer. Med Sci Sports Exerc 2004;36:1042-7.

16. Alexiou H, Coutts AJ. A comparison of methods used for quantifying internal training load in women soccer players. Int J Sports Physiol Perform 2008;3:320-30.

17. Borg GA. Psychophysical bases of perceived exertion. Med Sci Sports Exerc 1982;14:377-81.

18. Nakamura FY, Moreira A, Aoki MS. Monitoramento da carga de treinamento: a percepção subjetiva do esforço da sessão é um método confiável? Rev Educ Fís/UEM 2010;21(1)1-11.

19. Kelly VG, Coutts AJ. Planning and monitoring training loads during the competition phase in team sports. Strength Cond J 2007;29:32-7.

20. Giannichi RS. Medidas e avaliação em Educação Física. Viçosa: Imprensa Universitária, 1984.

21. Foster C, Heimann KM, Esten PL, Brice G, Porcari JP. Differences in perceptions of training by coaches and athletes. South African J Sports Med 2001;8:3-7.

22. Borresen J, Lambert MI. Quantifying training load: a comparison of subjective and objective methods. Int J Sports Physiol Perform 2008;3:16-30.

23. Wallace LK, Slattery KM, Coutts AJ. The ecological validity and application of the session-RPE method for quantifying training loads in swimming. J Strength Cond Res 2009;23:33-8.

\section{Endereço para correspondência}

Marcelo Saldanha Aoki

Universidade de São Paulo. Escola de Artes,

Ciências e Humanidades.

Grupo de Pesquisa em Adaptações Biológicas ao Exercício Físico.

Av. Arlindo Bettio, 1000 Ermelino Matarazzo. CEP: 03828-000. São Paulo, SP. Brasil

E-mail: saldanha.caf@usp.br 